\title{
Motion Correction of PET Images Using Multiple Acquisition Frames
}

\author{
Yani Picard* and Christopher J. Thompson, Member, IEEE
}

\begin{abstract}
Positron emission tomography (PET) is a relatively lengthy brain imaging method. Because it is difficult for the subject to stay still during the data acquisition, head motion during scans is a source of image degradation. A simple data acquisition technique to reduce the effect of this problem is described. The technique associates the incoming data with the real-space position of the head. During the PET scan, the head position is constantly monitored with two video cameras and compared to its initial position. Every time the displacement for a region within the field of view (FOV) is larger than a specified threshold displacement, the PET data acquisition system starts to save the PET data in a new frame. The total number of frames required for a complete study depends on the magnitude of the head motion during the study and on the threshold displacement. At the end of the study, all the acquired frames are reconstructed independently and each image is rotated and translated to coincide with the initial position. When these images are summed, they produce a final image with fewer motion artefacts.
\end{abstract}

Index Terms - Acquisition, motion correction, positron emission tomography, video monitoring.

\section{INTRODUCTION}

A $\mathrm{S}$ the resolution of positron emission tomography (PET) increases, image degradation due to subject movement becomes more evident. Currently, most PET centers use head restraints to reduce the head motion. Nevertheless, they do not prevent nodding movements [1], [2]. To monitor these movements, several video monitoring techniques for subjects undergoing PET studies have been reported ${ }^{1}$ [1], [3], [4]. While there are several algorithms to align images reconstructed from different PET studies [5], [6], only two techniques which correct for the image blurring due to head motion during studies have been reported [4], [7]. These techniques rebin the data in the sinograms by acquiring the data in list mode along with the head position [7] or by using a look-up table to select the appropriate sinogram location according to the head position in real time [4]. Although these

Manuscript received November 17, 1995; revised December 18, 1996. This work was supported by General Electric Medical Systems (Pet Engineering) and the Medical Research Council of Canada under Grant SP30. The Associate Editor responsible for coordinating the review of this paper and recommending its publication was M. W. Vannier. Asterisk indicates corresponding author.

*Y. Picard is with the Medical Physics Unit and Montreal Neurological Institute, McGill University, Montreal, P.Q. H3A 2B4 Canada (e-mail: yani@rclvax.medcor.mcgill.ca).

C. J. Thompson is with the Medical Physics Unit and Montreal Neurological Institute, McGill University, Montreal, P.Q. H3A 2B4 Canada.

Publisher Item Identifier S 0278-0062(97)02402-6.

${ }^{1}$ Brian Reece Scientific Ltd, Real time video subtraction system supplied to the MRC cyclotron unit, Hammersmith hospital, for patient position monitoring/recording, Advertising brochure, July 1992. techniques correct for motion, they require much disk space and they introduce normalization error since the data in the sinograms is likely to be multiplied by normalization factors corresponding to the wrong detector pairs.

This paper describes a simple technique to correct for motion blurring, in which multiple acquisition frames are used during a single study. During the study, the video-camerabased surveillance system described in [1] is used to monitor the head position at a rate of 15 images/s. The video system is now equipped with an input-output $(\mathrm{I} / \mathrm{O})$ card that is connected to an I/O interface wired to a Scanditronix PC-2048B [8] 15 Slice PET acquisition system. This interface has previously been used to acquire the data in evoked vascular response (EVR) mode [9] and allows the users to store the acquired data in an externally selectable region of memory. Eight frames that were initially designed for cardiac gated studies are now available at any time throughout the data acquisition period. If significant head motions are detected, the video system sends a signal setting up the acquisition system to record the data into a proper position related frame. A complete study is then acquired over several frames. The technique is explained in more detail in the Section II.

For this paper, the technique was tested for both discrete and continuous motion of different source objects. An evaluation of the displacement threshold that should be used for routine scans was also performed.

\section{METHODS}

The video camera system utilized consists of two chargedcoupled device (CCD) cameras placed on the gantry of the scanner. They are connected to a frame grabber via a screen splitter so that the images of the two cameras can be digitized simultaneously [1]. Motion measurements of the source in the scanner's field of view (FOV) (phantom or subject's head) are done by fixing three light emitting diodes (LED's) on the source such that they can be seen simultaneously by both cameras producing six bright spots on the image. The frame grabber continuously digitizes the incoming image and refreshes a $512 \times 480$-pixel frame buffer every $1 / 30 \mathrm{~s}$. The centroid of each bright spot is calculated by extracting from the frame buffer six small rectangular pixel matrices $(12 \times 12$ pixels or smaller depending on the LED size and their distance from the camera), each centered on the six centroids of the previous frame. After calibration (see below), the six bright spots centroids are converted to real-space positions of the LED's using triangulation. From rigid body motion theory [10], the positions of three independent point coordinates 
are sufficient to specify the body's position in space at any time. Furthermore, a rigid body transformation from an initial position (subscript $i$ ) to a final position (subscript $f$ ) is fully described by a rotation followed by a translation. For each particle position $(x, y, z)$ in the rigid body

$$
\left(\begin{array}{l}
x \\
y \\
z
\end{array}\right)_{f}=\mathbf{R}\left(\begin{array}{l}
x \\
y \\
z
\end{array}\right)_{i}+\left(\begin{array}{l}
t_{x} \\
t_{y} \\
t_{z}
\end{array}\right)
$$

where $\mathbf{R}$ is a $3 \times 3$ rotation matrix and $\left(t_{x}, t_{y}, t_{z}\right)$ is a translation vector. Each element of the rotation matrix $\mathbf{R}$ is a triginometric function of three rotation angles. A rigid body thus needs six parameters (three rotation angles and three translation coordinates) to describe the position of its particles after motion. Knowing the position in space of the three LED's at any time and assuming that the source is a rigid body, (1) results in an overdetermined system of nonlinear equations (nine equations for six unknowns). This system of nine equations is solved using the Newton-Raphson iterative method [11]. This method transforms the nonlinear system to a linear system which is solved using singular value decomposition (SVD) [11].

Since the publication of our previous paper [1], the position of the cameras has been modified to accommodate the technicians and researchers using the scanner. The cameras are now positioned on the top left and right corners of the gantry (Fig. 1). Because of this new configuration, the calibration of the video system now consists of determining six extrinsic parameters (three for the position and three for the orientation of the camera) with respect to the coordinate system of the scanner, and five intrinsic parameters (the focal distance, the radial distortion parameter, two parameters for the lens center pixel coordinate, and a scale factor due to camera scanning and acquisition timing error) for each camera. These parameters are computed in two stages using the technique described in [12] for calibrating a camera using monoview noncoplanar points. These noncoplanar points are obtained by moving an LED to at least 25 different positions in the FOV of the cameras using an XY translation stage (Compumotor Corporation, Petaluma, CA) controlled by the video system computer via an RS232 interface, and the up-down motion of the scanner couch. The resulting systems of linear equations are solved using SVD.

The cameras' right-hand orthogonal coordinate system shown in Fig. 1 was used to describe the motion of the source objects: The $x$-axis and $y$-axis define a plane parallel to the scanner slices. The $z$-axis is the scanner axis. When the tilt of the gantry is set to $0^{\circ}$ so that the couch is orthogonal to the scanner rings, motion along the $x$-axis corresponds raising the scanner couch. Motion along the $y$-axis corresponds to a lateral displacement of the couch from left to right. The origin of the coordinate system is in the center of slice 8 of the scanner (middle slice). The cameras and their coordinate system are relative to the gantry, i.e., a tilt of the gantry tilts the cameras' coordinate system about its $y$-axis.

The video system is controlled by a MicroVAX II which is part of a VMS cluster [1]. For the following studies, the MicroVAX II was modified by installing a parallel I/O card

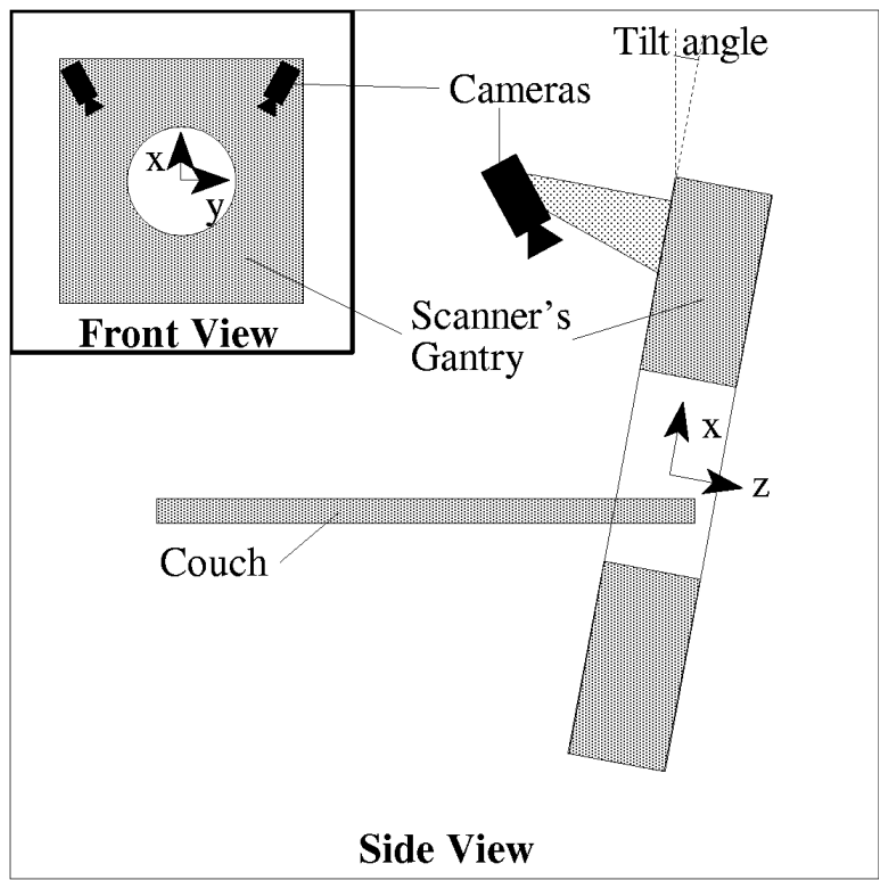

Fig. 1. Side view of the scanner showing the cameras' position and the right-hand orthogonal coordinate system of the camera system. The inset shows the cameras' position from a front view of the gantry.

and cable to connect it to a modified programmable read-only memory (PROM) board (used for EVR studies) [9] located in the scanner acquisition system. The PROM board converts the crystal identification pair information into a sinogram address. It also selects the base memory address of the sinogram buffers during cardiac gated studies. With these modifications in place, the video system can continuously send a four bit signal to the modified PROM board to select the memory buffer where the acquired data should be stored. Eight different memory buffers are available and can be selected via the video system by sending numbers between one $\left(0001_{2}\right)$ and eight $\left(1000_{2}\right)$ to the parallel I/O card. Sending zeroes $\left(0000_{2}\right)$ will force the data acquisition system to behave as if the normal PROM board was installed.

Fig. 2 shows the flow chart corresponding to the use of multiple acquisition frames during a normal PET study when the video system is monitoring the source or subject position and the communication with the data acquisition system is enabled. After setting the variables FRAME and FRAME_USED to one, the data acquisition system starts the scan and the video system records the initial position associated with the current frame (at this point the current frame is frame 1). The video system also records the current time and frame number in a frame history file (this file is used after completion of the scan to correct for decay as explained in the next paragraph). The data is collected in this frame until the displacement detected by the video system is larger than a specified threshold (box 8). When this happens, the video system checks whether a previously selected frame has an associated initial position which is close to the current position (boxes 10,12, 13). If so, this frame is selected and the video system records the current time and frame number in the frame history file; If not, a new 


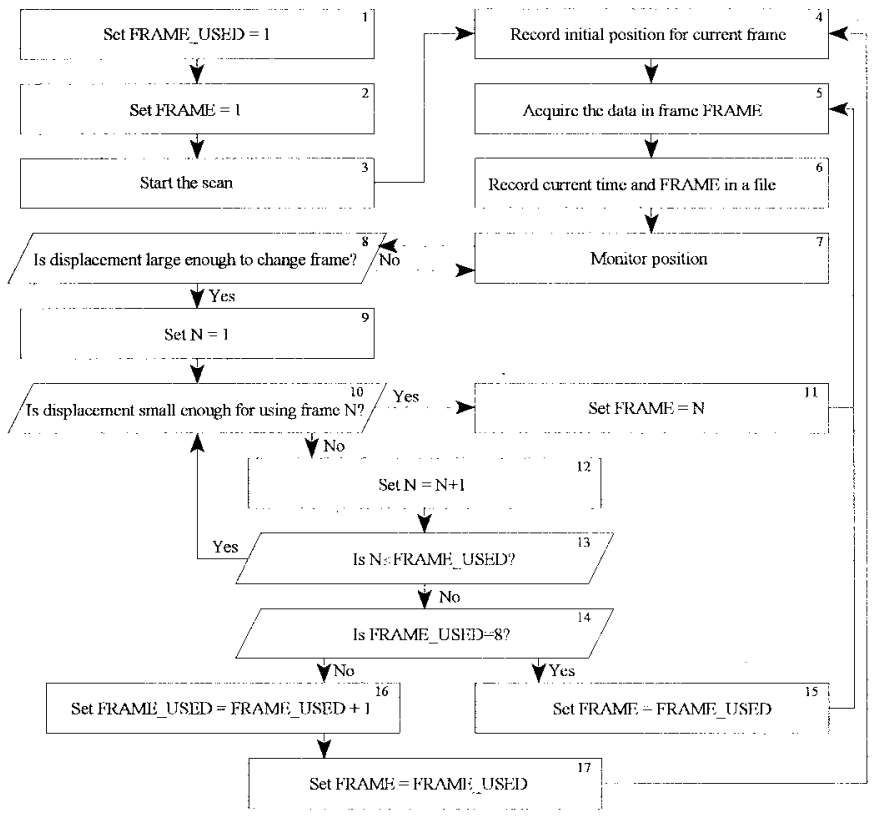

Fig. 2. Flow chart of the acquisition technique. The variable FRAME is sent by the video system computer to the acquisition system.

acquisition frame is requested and the initial (current) position for this new frame is recorded along with the current time and frame number. Box 14 limits the number of frames to eight by forcing the data to be stored in frame 8 if more than eight frames are required during a single scan. After the acquisition, the frames are reconstructed independently and corrected for decay (see below), and each image is rotated and translated according to its associated initial position. The images are then added together.

Because the frames can be visited more than once, the decay correction is a bit more complex than usual. At the end of the scan, each frame is corrected for decay using the relationship

$$
\begin{aligned}
f_{\text {decay }} & =\frac{\sum_{i=1}^{N} \Delta t_{i}}{\sum_{i=1}^{N} \int_{t_{i}}^{t_{i}+\Delta t_{i}} e^{-\ln 2 t / T_{1 / 2}} d t} \\
& =\frac{\ln 2}{T_{1 / 2}} \frac{\sum_{i=1}^{N} \Delta t_{i}}{\sum_{i=1}^{N} e^{-\ln 2 t_{i} / T_{1 / 2}}\left(1-e^{\left.-\ln 2 \Delta t_{i} / T_{1 / 2}\right)}\right.}
\end{aligned}
$$

where $f_{\text {decay }}$ is the decay factor for one particular frame. $N$ is the number of times the frame was visited. $t_{i}$ and $\Delta t_{i}$ are the times and time intervals during which the frame was visited. These parameters are extracted from the frame history file. $T_{1 / 2}$ is the half-life of the isotope used. This equation is reduced to the usual decay correction factor

$$
f_{\text {decay }}=\frac{\ln 2 \Delta t}{T_{1 / 2}} \frac{e^{\ln 2 t / T_{1 / 2}}}{1-e^{-\ln 2 \Delta t / T_{1 / 2}}}
$$

when a particular frame is visited only once (i.e., when $N=1$ ) at time $t$ for a duration of $\Delta t$.

\section{A. Study of Discrete Motion on Line Source Images}

As an initial measure of the system performance, this technique was tested using the discrete motion of a line source.
TABLE I

Relative Hoffman Phantom Positions

\begin{tabular}{||c|c|c|c|c||}
\hline \hline Position & $\begin{array}{c}\text { Couch } \\
\text { UP } \\
(\mathrm{mm})\end{array}$ & $\begin{array}{c}\text { Couch } \\
\text { IN } \\
(\mathrm{mm})\end{array}$ & $\begin{array}{c}\text { Gantry } \\
\text { Tilt } \\
\left({ }^{\circ}\right)\end{array}$ & $\begin{array}{c}\text { Phantom } \\
\text { Rotation } \\
\left({ }^{\circ}\right)\end{array}$ \\
\hline \hline 1 & 0.0 & 6.0 & 0 & 0 \\
2 & 1.9 & 0.0 & 0 & 0 \\
3 & 4.1 & 6.0 & 0 & 0 \\
4 & 4.1 & 9.0 & 0 & 0 \\
5 & 4.1 & 6.0 & 4 & 0 \\
6 & 1.9 & 6.0 & 0 & 4 \\
7 & 1.8 & 3.0 & 1 & 4 \\
\hline
\end{tabular}

The line source used was the same ${ }^{68} \mathrm{Ge}$ rod currently used for transmission scans (Activity $=1.5 \mathrm{mCi}$ ). The source was placed in the $x-y$ plane (parallel to the scanner slices) on an XYZ translation stage. Three LED's were also placed on the translation stage to compare the relative position of the transmission source to its original position. The gantry was tilted to simulate rotation of the source about the $y$-axis. With this setup, the following two experiments were conducted.

1) Translation study: Using the scanner laser, the line source was placed in slices 7-9 of the scanner (i.e., in increments of $6.5 \mathrm{~mm}$ along the $z$-axis).

2) Rotation study: Using the scanner laser, the line source was approximately centered in slice 1 of the scanner and the gantry was tilted to $-5^{\circ}, 0^{\circ}$, and $+5^{\circ}$.

For each of the positions described in the two studies, the position of the line source was recorded using the video system. Thirty-second scans were performed at each position using three different frames. Each frame was reconstructed individually using a 2-mm Hanning filter without correction for attenuation. In both studies, the final image resulting from a simple sum of the images in each frame was compared to the final image produced by adding the images rebinned according to the position data.

\section{B. Study of Discrete Motion on Hoffman Phantom Images}

Three LED's were placed around the based circumference (at about $60^{\circ}$ intervals) on the most anterior section of the cylinder containing the Hoffman brain phantom [13]. The phantom was filled with $10 \mathrm{mCi}$ of ${ }^{18} \mathrm{~F}-\mathrm{FDG}$. The phantom was scanned for $10 \mathrm{~min}$ in each of the seven positions listed in Table I. One frame was acquired per position. To simulate nodding movement, the gantry was tilted to different angles instead of rotating the phantom about the $y$-axis. This permits a configuration similar to the nodding motion of the phantom without displacing the air bubbles that were trapped in the phantom. It is possible to record a nodding movement with this configuration only because the video system is attached to the gantry and tilts with it. $5 \mathrm{~h}$ later, when the activity in the phantom was $1.5 \mathrm{mCi}$, a 1 -h transmission scan was performed with the phantom in the sventh position. The seven emission scans were reconstructed individually using a 7-mm Hanning filter. The images were corrected for decay using (2). The original transmission scan (without registration onto the 


\section{Hoffman Phantom}

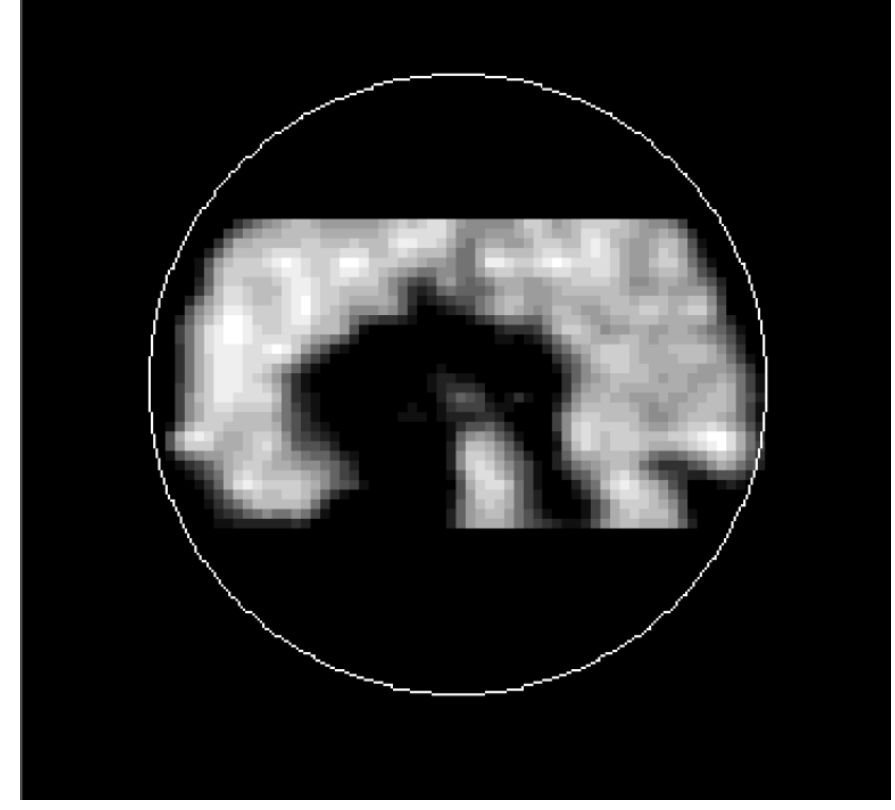

Fig. 3. Lateral PET image showing the region (within the circle) where the displacement had to be less than the threshold displacement to avoid blurring of the source due to motion. The image was acquired by filling the Hoffman phantom with $10 \mathrm{mCi}$ of ${ }^{18} \mathrm{~F}$-FDG and scanning for $10 \mathrm{~min}$ without motion. This image also corresponds to the image of the first of the seven frames in the study of discrete motion on Hoffman phantom.

emission data) was used for the attenuation correction of each scan. The reason for using the original transmission scans is given in the results and discussion section.

The seven images were first summed without any correction for motion. The frames were then registered according to the measured phantom positions and were subsequently summed. The two images were finally compared to a static image by selecting two regions of interest (ROI's) in slice 4 (ROI's A and B) and two others in slice 10 (ROI's C and D). These ROI's are shown in Fig. 6 of the next section.

\section{Study of Continuous Motion on Line Source Images}

Since nodding motion is the most frequent motion of the head during a scan [2], the effect of continuous nodding (about the $y$-axis) of a line source on PET images was investigated. A normalization $\mathrm{Ge}^{68}$ rod (activity $=650 \mu \mathrm{Ci}$ ) was positioned along the scanner axis with the gantry tilt set to $0^{\circ}$. One LED was attached to the tip of the line source. This single reference point was sufficient to describe the motion of the source in time since the rod was limited to a single degree of freedom, that is a rotation about the $y$-axis. The extent of the source was larger than the scanner axial FOV. The source was scanned for five scans of $60 \mathrm{~s}$ while moving continuously the gantry back and forth between tilt angles $-A_{\max } / 2$ and $+A_{\max } / 2$ for each scan. The values used for the total angular displacement $A_{\max }$ are summarized in Table II. By entering the value of $A_{\max }$ into the software written to control the video system I/O card prior scanning, the video system could divide the total angular displacement into eight subregions. The data acquired by the scanner was then stored in the
TABLE II

Angle Swept By Gantry Tilt and Corresponding Values

\begin{tabular}{||c|c|c|c||}
\hline Scan number & $\mathrm{A}_{\max }$ & $\begin{array}{c}\text { Subregion } \\
\text { extent } \\
\left({ }^{\circ}\right)\end{array}$ & $\begin{array}{c}\text { Total } \\
\text { displacement } \\
\text { in slice 1 } \\
(\mathrm{mm})\end{array}$ \\
\hline \hline 1 & $\left({ }^{\circ}\right)$ & 4 & 26.1 \\
2 & 32 & 3 & 19.3 \\
3 & 16 & 2 & 12.8 \\
4 & 8 & 1 & 6.4 \\
5 & 4 & 0.5 & 3.1 \\
\hline
\end{tabular}

memory locations corresponding to the subregion location of the source by sending the proper 4-b signals to the PROM board. The extent of the subregions are listed in Table II. Table II also contains the total displacement of the line source in slice 1 . The total displacement in slice 8 is $0 \mathrm{~mm}$ because the gantry is tilted about the $y$-axis which is in slice 8 . The total displacement in the other slices can be calculated by interpolation.

After the study, each frame was reconstructed individually using a 2-mm Hanning filter without correcting for attenuation. A final image resulting from a simple sum of the images in each frame was compared to a final image produced by adding the images rebinned according to the position data.

\section{Study of Continuous Motion on Hoffman Phantom Images}

One LED was placed on the anteriormost section of the base circumference of the cylinder containing the Hoffman brain phantom. This single reference point was sufficient to describe the motion of the source in time since the phantom was limited to a single degree of freedom, that is a rotation about the $y$ axis. The phantom was filled with $1.4 \mathrm{mCi}$ of ${ }^{18} \mathrm{~F}-\mathrm{FDG}$. The phantom was scanned for $30 \mathrm{~min}$ while continuously moving the gantry to different positions to simulate nodding motion. The multiple frame acquisition technique described in Fig. 2 was used. A cylindrical region in the FOV (circle of Fig. 3) where the displacement had to be always smaller than the threshold displacement was defined to reduce the blurring of the object due to nodding motion. Because the nodding is about the $y$-axis, the maximum displacement always occurred on the boundary of the region. The initial gantry position was $0^{\circ}$ associated with frame 1. A day later, a transmission scan was performed. The images were reconstructed separately using a 7-mm Hanning filter and corrected for decay.

The images were first summed without any correction for motion. The frames were then registered according to the measured phantom positions and were subsequently summed. The two resulting images were finally compared by selecting two ROI's (ROI's E and F shown on Fig. 9).

\section{RESUlTS AND DiscusSION}

\section{A. Study of Discrete Motion on Line Source Images}

The images in Fig. 4 were obtained for the discrete translation study. The top row corresponds to slices 6-10 of the 


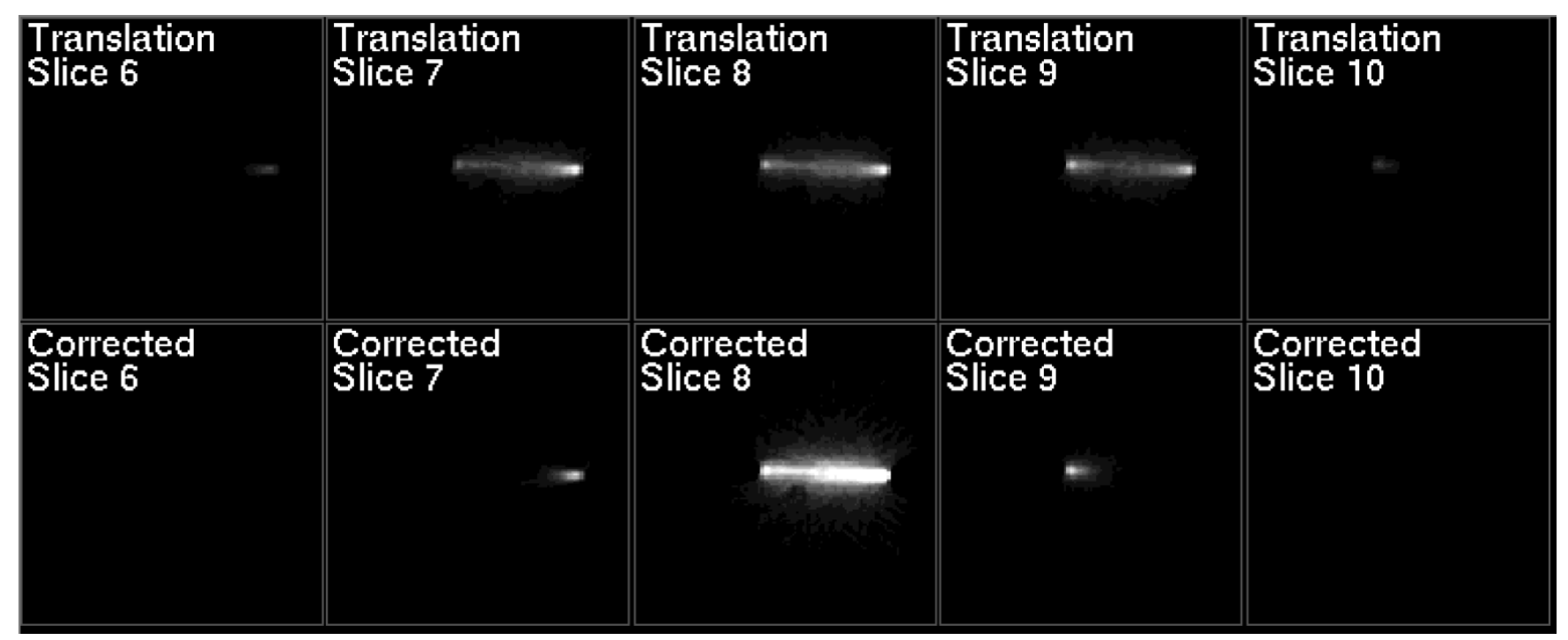

Fig. 4. (top row) Effect of discrete translation of a line source in planes 7, 8, and 9; (bottom row) Images corrected for discrete translations. The grayscale used is the same for all slices.

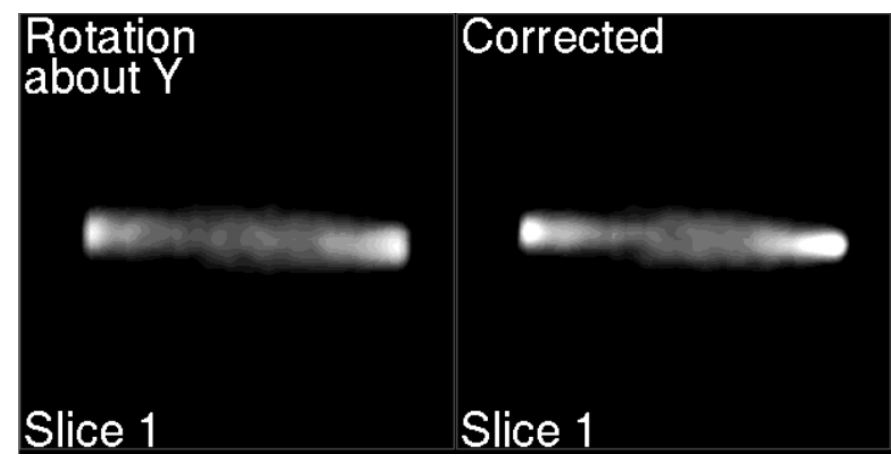

Fig. 5. Effect of rotation about the $y$-axis for a line source in slice 1 . The image is $128 \times 128$ pixels. The pixel width is $2 \times 2 \mathrm{~mm}$.

uncorrected images. The intensity along the line source varies within slices since the source was not placed exactly parallel to the $x-y$ plane. In fact, when the source was scanned in plane $\mathrm{N}(N=7,8$, or 9$)$ the left extremity of the rod was in plane $N+1$ and the right extremity in plane $N-1$. Nevertheless, the intensity in the center of the rod is equal for slices 7-9.

The bottom row shows the resulting image after correcting for motion. The intensity of the line source is about three times greater than the intensity in slices $7-9$ of the top row. Both rows are normalized to the same grayscale.

Fig. 5 was obtained for the discrete rotation about the $y$-axis. Both images are normalized to the same grayscale. The image is slightly less blurred in the corrected image. The difference between the images is small since a rotation of $5^{\circ}$ about the $y$-axis corresponds to a displacement of about $4 \mathrm{~mm}$ in slice 1 (which is $45.5 \mathrm{~mm}$ away from the $z=0$ position). The resolution of the scanner is 5-6 $\mathrm{mm}$ [8].

\section{B. Study of Discrete Motion on Hoffman Phantom Images}

Fig. 6 are the resulting images of the Hoffman phantom scans for slice 4 and 10. All images are displayed using the grayscale located at the center of the figure. The left side of the figure shows the resulting images of the Hoffman phantom

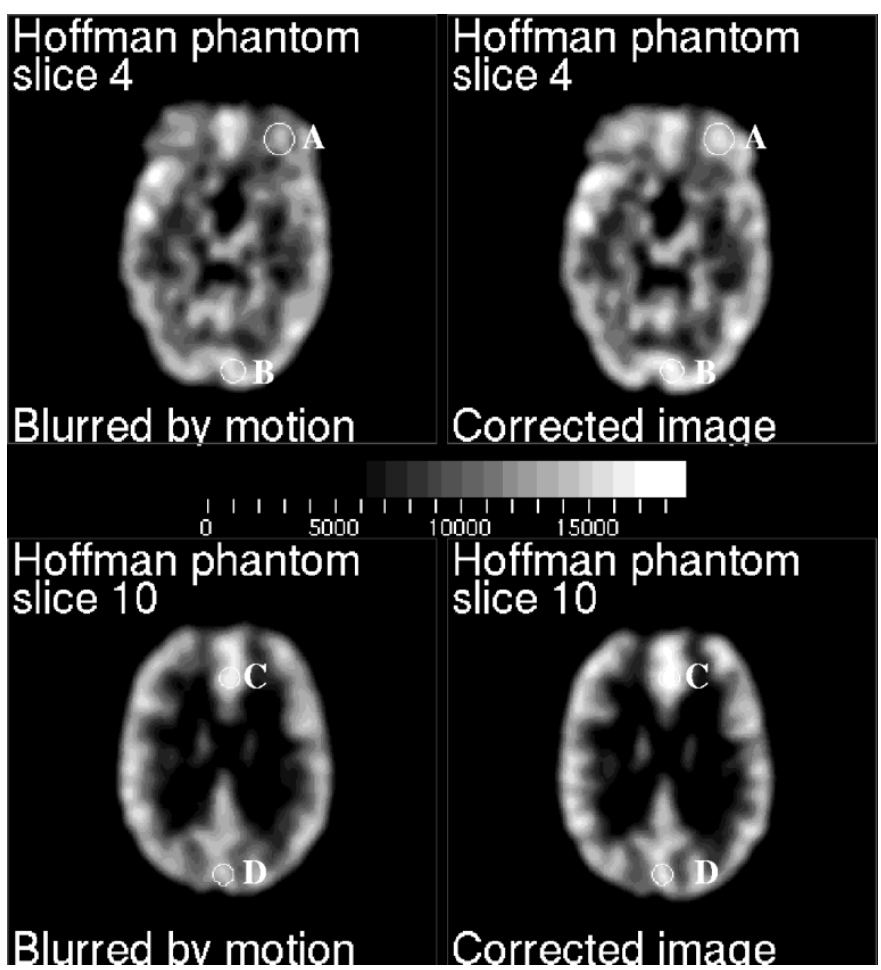

Fig. 6. Effect of discrete motion on Hoffman phantom. The image is $128 \times 128$ pixels. The pixel width is $2 \times 2 \mathrm{~mm}$.

blurred by the discrete motion. The images on the right were produced after correcting the emission scan for motion but using the original (unmoved) transmission scan. The images on the right side look sharper than those on the left side. The maxima for the images on the right are greater (brighter) than those on the left. To evaluate the difference in these maxima, two ROI's on two relative maxima in slice 4 (ROI's A and B) and two others in slice 10 (ROI's C and D) were studied. The maximum displacements of the ROI's when the Hoffman phantom was moved to the seven different positions were 9.5, $11.4,9.3$, and $11.0 \mathrm{~mm}$ for ROI's A-D, respectively. 
TABLE III

ROI Measurements on Hoffman Phantom

\begin{tabular}{||c|c|c|c|c|c|c||}
\hline \multirow{2}{*}{ ROI } & \multicolumn{2}{|c|}{ Static } & \multicolumn{2}{c||}{ Blurred by motion } & \multicolumn{2}{c||}{ Corrected } \\
\cline { 2 - 7 } & $\begin{array}{c}\text { Mean } \\
(\mu \mathrm{Ci} / \mathrm{cc})\end{array}$ & $\begin{array}{c}\text { Std } \\
(\mu \mathrm{Ci} / \mathrm{cc})\end{array}$ & $\begin{array}{c}\text { Mean } \\
(\mu \mathrm{Ci} / \mathrm{cc})\end{array}$ & $\begin{array}{c}\text { Std } \\
(\mu \mathrm{Ci} / \mathrm{cc})\end{array}$ & $\begin{array}{c}\text { Mean } \\
(\mu \mathrm{C} / \mathrm{ccc})\end{array}$ & $\begin{array}{c}\mathrm{Std} \\
(\mu \mathrm{C} / \mathrm{cc})\end{array}$ \\
\hline \hline $\mathrm{A}$ & 15.0 & 0.94 & 12.3 & 1.08 & 14.6 & 0.84 \\
$\mathrm{~B}$ & 15.9 & 1.39 & 14.7 & 0.84 & 15.9 & 1.02 \\
$\mathrm{C}$ & 17.3 & 0.83 & 16.0 & 0.70 & 16.9 & 0.68 \\
$\mathrm{D}$ & 14.2 & 1.20 & 13.6 & 1.01 & 14.6 & 1.25 \\
\hline
\end{tabular}

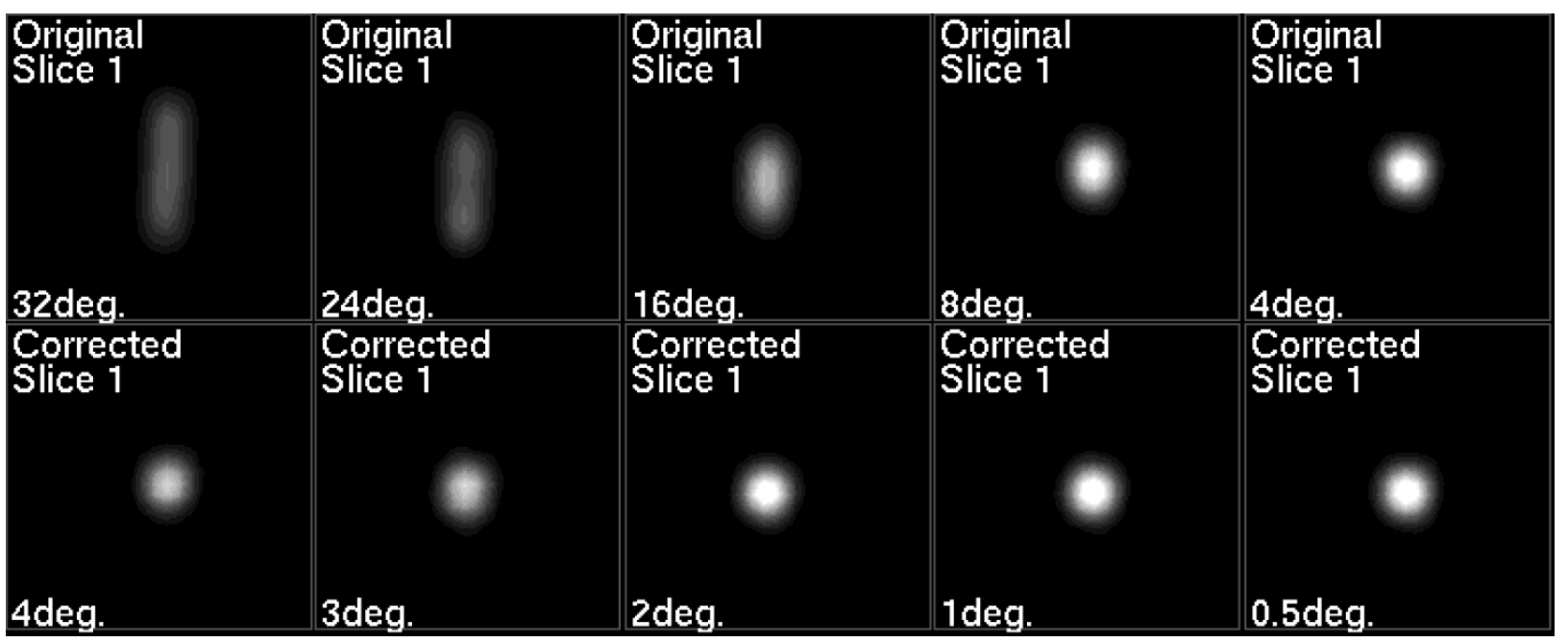

Fig. 7. Effect on slice 1 caused by continuous rotation of a line source about the $y$-axis. (top row) Original blurring effect caused by continuous rotation of the line source (obtained by tilting the gantry) between $-A_{\max } / 2$ and $+A_{\max } / 2 . A_{\max }$ is indicated in the bottom left corner of the images. (bottom row) Resulting images obtained by rebinning the eight frames of each image of the top rowand limiting the blurring to the angle specified in the bottom left corner of the images which correspond to $A_{\max } / 8$.

Table III lists the ROI measurements (mean and standard deviations) on a static image, on the blurred image, and on the motion-corrected images. For the static image, the first frame was normalized by multiplying the pixels by seven (after correction for decay). The ROI's were decreased by $18.0 \%$, $7.5 \%, 7.5 \%$, and $4.2 \%$ for ROI's A-D, respectively, due to motion. By rebinning the images according to their position, the ROI's were closer to the static image ROI's (off by $-2.6 \%$, $0.0 \%,-2.3 \%$, and $+2.8 \%$, respectively, from ROI's in the static image). These represent an increase from the blurred image by $18.7 \%, 8.2 \%, 5.6 \%$, and $7.4 \%$, respectively.

These results are significant if one considers that the maximum displacement was only about $1 \mathrm{~cm}$ for all ROI's and the Hoffman phantom activity distribution was a relatively smooth distribution. They indicate the need to compensate for motion by rebinning the images according to the source position when the displacement of at least one point in the source is $1 \mathrm{~cm}$ or more from its initial position.

The attenuation correction would be more accurate if the transmission scan and the emission scans were registered using the video system, but to date this has not been implemented. The main difficulty anticipated in implementing this registration arises from the fact that parts of the attenuating media (i.e., the couch and the head restraint) within the FOV are not necessarily moving with the source. Some pattern recognition and image segmentation would be required to register the transmission and emission scans accurately according to the motion of the source.

\section{Study of Continuous Motion on Line Source Images}

The top row of Fig. 7 shows the effect on slice 1 caused by the continuous rotation of a line source about the $y$-axis for different sweep angles. In this case, the eight frames acquired per experiment were simply added together. The bottom row of Fig. 7 illustrates the improvement in the images when the frames are rebinned after reconstruction. All the images were improved by acquiring the data in eight different frames instead of a single frame. The improvement on the rightmost images is negligible. Thus, the effect of changing the frame every $0.5^{\circ}$ results in a large number of frames being requested from the PET data acquisition hardware without a significant improvement in the corrected image. A visible improvement in the images was seen when the sweep angle was $8^{\circ}$ or more. This sweep angle corresponds to a total displacement of 6.4 $\mathrm{mm}$ along the $y$-axis of the image (see Table II). Considering that the data acquisition of our scanner has a limit of eight frames per study and that the activity distribution of a routine head scan is smoother than the line source used here, a good threshold displacement to switch the frame would correspond 
TABLE IV

InFormation About EACH ACQUisition Frame

\begin{tabular}{||c|c|c|c||}
\hline $\begin{array}{c}\text { Frame } \\
\text { number }\end{array}$ & $\begin{array}{c}\text { Total } \\
\text { Acquisition } \\
\text { Time } \\
(\mathrm{s})\end{array}$ & $\begin{array}{c}\text { Number of } \\
\text { Intervals }\end{array}$ & $\begin{array}{c}\text { Decay } \\
\text { Correction } \\
\text { Factor }\end{array}$ \\
\hline \hline 1 & 186.7 & 19 & 1.1071 \\
2 & 256.5 & 21 & 1.1081 \\
3 & 232.3 & 22 & 1.0968 \\
4 & 159.4 & 10 & 1.0774 \\
5 & 298.7 & 18 & 1.1039 \\
6 & 119.3 & 18 & 1.1180 \\
7 & 269.0 & 18 & 1.1050 \\
8 & 277.8 & 9 & 1.0798 \\
\hline
\end{tabular}

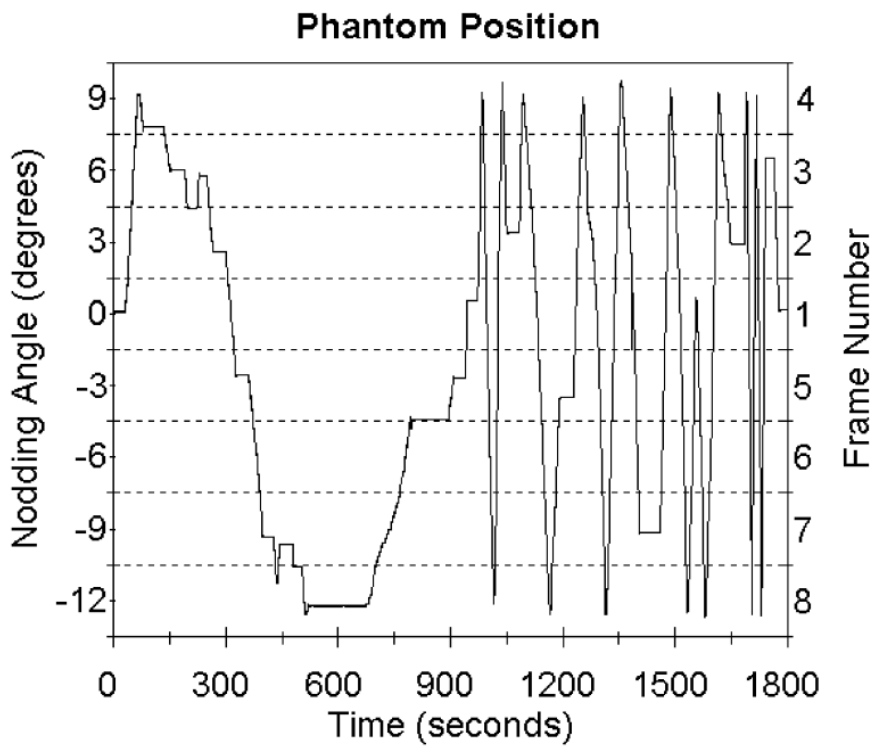

Fig. 8. Plot of the Hoffman phantom nodding angle as a function of time The corresponding acquisition frame can be found on the right side of the graph.

to a displacement between $3.1 \mathrm{~mm}\left(A_{\max }=4^{\circ}\right)$ and 6.4 $\mathrm{mm}$. It would then be reasonable to use this technique with a threshold displacement corresponding to the resolution of the scanner for routine scans, that is $5 \mathrm{~mm}$ [8]. If the images are reconstructed using a smoothing filter which produces a final image resolution worse than $5 \mathrm{~mm}$, the threshold could be set coarser.

\section{Study of Continuous Motion on Hoffman Phantom Images}

From the conclusion of the previous experiment, a threshold displacement of $5 \mathrm{~mm}$ was determined. Thus, when the video system detected a displacement larger than $5 \mathrm{~mm}$ along the circle of Fig. 3, a signal was sent to the acquisition system to change the acquisition frame.

Fig. 8 shows the nodding angle as a function of time for this study. The scan was started using frame 1 with a nodding angle of $0^{\circ}$. The nodding angle was then increased rapidly to $9^{\circ}$ requiring the data to be recorded in frames -4 . At this point

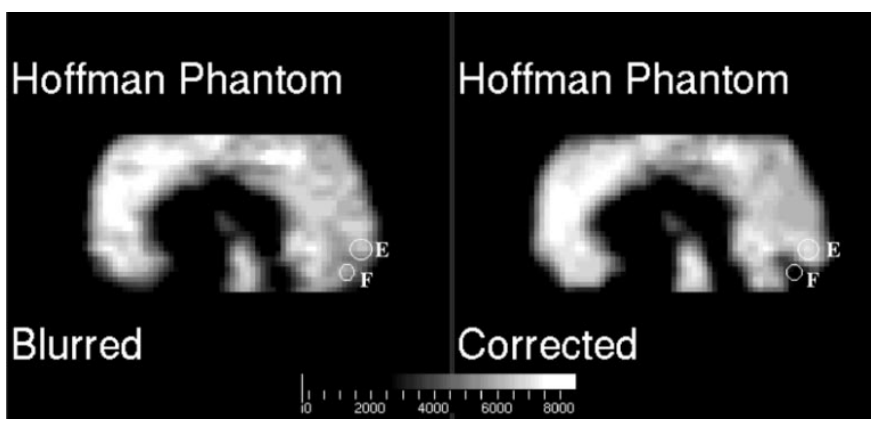

Fig. 9. Effect of Hoffman phantom nodding motion on a lateral PET image. (a) Original blurring effect caused by continuous nodding motion of Hoffman phantom as plotted in Fig. 8. (b) Corrected image using multiple acquisition frame and correcting for motion.

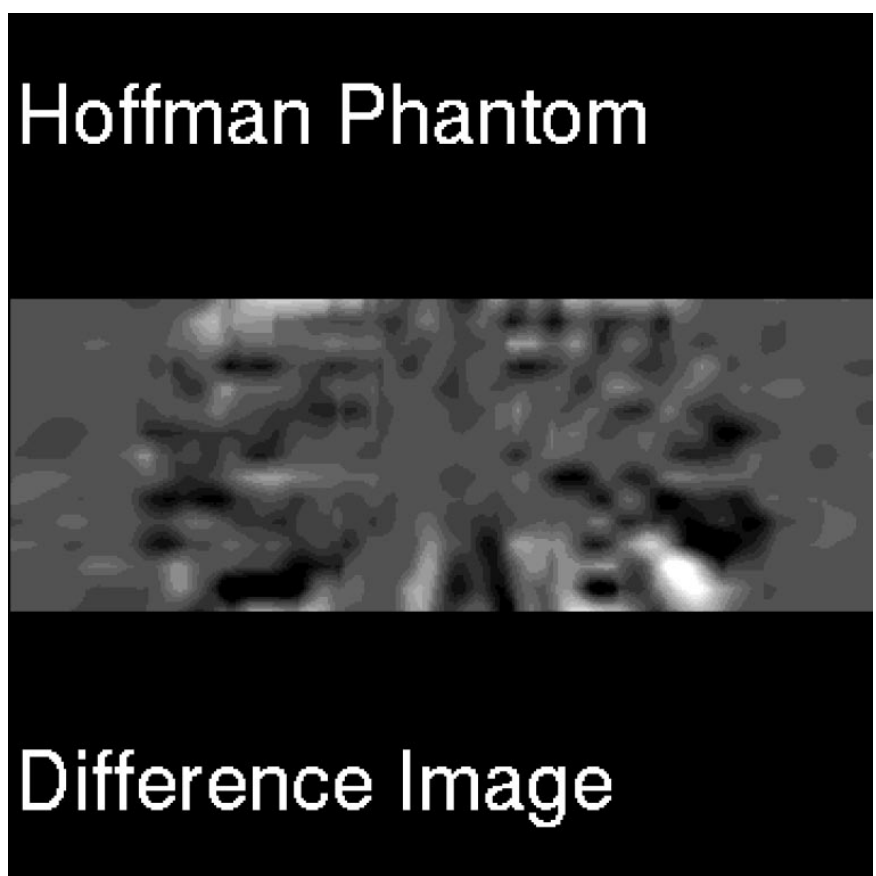

Fig. 10. Subtraction of the blurred image by the corrected image for the study of continuous nodding motion. Both images are shown in Fig. 9. Black corresponds to a negative difference and white to a positive difference. A mid-gray color fills the regions were the difference was negligible.

the angle started to decrease forcing the data to be recorded back to frame 3,2 , and 1 . The angle continued to decreased below $0^{\circ}$, forcing the video system to require new frames (frames 5-8). For the rest of the study, the nodding angle was moved continuously between $-12^{\circ}$ and $9^{\circ}$ to limit the acquisition to eight frames. Table IV shows some information about each acquisition frame. The data was extracted from the frame history file. The total acquisition time and the number of intervals could also be computed from a graph similar to that shown in Fig. 8. The decay correction factor was computed using (2) $\left(T_{1 / 2}=6582 \mathrm{~s}\right.$ for $\left.{ }^{18} \mathrm{~F}\right)$. Notice that the decay factors are all about the same since the frames were not selected in sequence but according to the phantom position which was changed continuously during the study.

Fig. 9 shows the images resulting from this experiment. The image corrected for motion is on the right. This image 
TABLE V

ROI Measurements on Hoffman Phantom Nodding

\begin{tabular}{||c|c|c|c|c|c|c||}
\hline \multirow{2}{*}{ ROI } & \multicolumn{2}{|c|}{ Static } & \multicolumn{2}{c|}{ Blurred by nodding } & \multicolumn{2}{c||}{ Corrected } \\
\cline { 2 - 7 } & $\begin{array}{c}\text { Mean } \\
(\mathrm{nCi} / \mathrm{cc})\end{array}$ & $\begin{array}{c}\text { Std } \\
(\mathrm{nCi} / \mathrm{cc})\end{array}$ & $\begin{array}{c}\text { Mean } \\
(\mathrm{nCi} / \mathrm{cc})\end{array}$ & $\begin{array}{c}\text { Std } \\
(\mathrm{nCi} / \mathrm{cc})\end{array}$ & $\begin{array}{c}\text { Mean } \\
(\mathrm{nCi} / \mathrm{cc})\end{array}$ & $\begin{array}{c}\text { Std } \\
(\mathrm{nCi} / \mathrm{cc})\end{array}$ \\
\hline \hline $\mathrm{E}$ & 2552 & 684 & 4902 & 436 & 3132 & 774 \\
$\mathrm{~F}$ & 7032 & 596 & 5374 & 348 & 6499 & 597 \\
\hline
\end{tabular}

is less blurred than the one on the left and approaches the image of Fig. 3 acquired without any motion. Table V lists the two ROI's measurements on the static image of Fig. 3 (ROI's are not shown) and on the two images shown in Fig. 9. ROI E corresponds to a hot spot and ROI F to a cold spot. The motion causes ROI E to increase by $92.1 \%$ and ROI $\mathrm{F}$ to decrease by $23.6 \%$. After correction for motion, ROI E was increased by $22.3 \%$ and ROI $\mathrm{F}$ was reduced by $7.6 \%$, compared to the original image. This represents an improvement of $36.1 \%$ for ROI E and of $20.9 \%$ for ROI F from the blurred image. An other way to appreciate the result is shown in Fig. 10 where the two images on Fig. 9 were subtracted. Since the nodding corresponded to a rotation about the $y$-axis of the scanner, that is the center of the pixel matrices of Fig. 9, the motion correction is negligible in the center of the image. As the distance from the center of the image increases, the corrections become more significant.

\section{CONCLUSION}

A simple technique for the correction of head motion artifacts in PET images has been described. The technique is based on monitoring the head position using a video camera system and coupling the position with multiple dataacquisition frames. Improvements in the images were seen when this technique was used to correct for the discrete motion of a line source and for the discrete motion of a brain phantom. Correction for the continuous nodding of a line source or a Hoffman brain phantom about an axis parallel to the scanner slices showed improvements in the final images when the multiple frame acquisition technique is used. After analyzing the results, it was determined that the video system should send a signal to change the acquisition frame whenever any point in the frame is displaced by more than $5 \mathrm{~mm}$ from its original position.

\section{ACKNOWLEDGMENT}

The authors wish to thanks J. J. Moreno-Cantu for his help during the scans involving the Hoffman phantom.

\section{REFERENCES}

[1] Y. Picard and C. J. Thompson, "Digitized video subject positioning and surveillance system for PET," IEEE Trans. Nucl. Sci., vol. 42, pp. 1024-1029, 1995.

[2] M. V. Green, J. Seidel, S. D. Stein, T. E. Tedder, K. M. Kempner, C. Kertzman, and T. A. Zeffiro, "Head movement in normal subjects during simulated PET brain imaging with and without head restraint," J. Nucl. Med., vol. 35, pp. 1538-1546, 1994.

[3] S. R. Goldstein, M. E. Daube-Witherspoon, and M. V. Green, "A device for continuous measurement of head position during PET brain imaging," J. Nucl. Med., vol. 35, p. 40P, 1994.

[4] M. Menke, M. S. Atkins, and K. R. Buckley, "Compensation methods for head motion detected during PET imaging," IEEE Trans. Nucl. Sci., vol. 43, pp. 310-317, 1996.

[5] R. P. Woods, S. R. Cherry, and J. C. Mazziotta, "Rapid automated algorithm for aligning and reslicing PET images," J. Comput. Assist. Tomogr., vol. 16, pp. 620-633, 1992.

[6] M. A. Mintun and K. S. Lee, "Mathematical realignment of paired PET images to enable pixel-by-pixel substraction," J. Nucl. Med., vol. 31, p. 816, 1990.

[7] M. E. Daube-Witherspoon, Y. C. Yan, M. V. Green, and R. E. Carson, "Correction for motion distortion in PET by dynamic monitoring of patient position," J. Nucl. Med., vol. 31, p. 816, 1990.

[8] A. C. Evans, C. J. Thompson, S. Marrett, and E. Meyer, "Performance evaluation of the PC-2048: A new 15-slice encoded-crystal PET scanner for neurological studies," IEEE Trans. Med. Imag., vol. 10, pp. 90-98, 1991.

[9] B. Wang, C. J. Thompson, and M. Mazza, "Measurement of evoked vascular response to cerebral activation with PET," in Conf. Rec. 1994 IEEE Nuclear Science Symp., Medical Imaging Conf., Nov. 1994, pp. $1421-1423$.

[10] H. Goldstein, Classical Mechanics, 2nd ed. Reading, MA: AddisonWesley, 1980.

[11] W. H. Press, B. P. Flannery, S. A. Teukolsky, and W. T. Vetterling, Numerical Recipes: The Art of Scientific Computing, Cambridge, U.K.: Cambridge Univ., 1986.

[12] R. Y. Tsai, "A versatile camera calibration technique for high-accuracy 3D machine vision metrology using off-the-shelf TV cameras and lenses," IEEE J. Robot. Automat., vol. RA-3, pp. 323-344, 1987.

[13] E. J. Hoffman, D. Cutler, W. M. Digby, and J. C. Mazziotta, "Phantom to simulated cerebral blood flow and metabolic images for PET," IEEE Trans. Nucl. Sci., vol. 37, pp. 616-620, 1990. 